\title{
Estudio sobre los diferentes tiempos de acondicionamiento previo al sistema adhesivo universal en dentina de dientes de bovino
}

\begin{abstract}
Comparative study in vitro on the different times of conventional acid etching system before bonding with an universal adhesive on bovine dentina
\end{abstract}

Miguel Angel Saravia Rojas ${ }^{1, a}$, Allison Ruth Bernal Ponce ${ }^{2, b}$,

\section{RESUMEN}

Los adhesivos universales y su versatilidad con las técnicas adhesivas desatan una controversia entre el uso y no uso de ácido grabador antes de la aplicación del adhesivo universal. Objetivo: Encontrar el tiempo óptimo de grabado ácido previo a la aplicación del Clearfil Universal Bond sobre la dentina de diente de bovino, mediante el análisis de los valores de resistencia a la microtracción. Material y métodos: Estudio experimental, prospectivo y comparativo. Se utilizaron 6 incisivos centrales superiores de bovino, de los que se obtuvieron 80 especímenes separados en 4 grupos de 20 especímenes cada uno. Luego de cumplir con los parámetros de cada grupo, se sometió a los especímenes a la prueba de microtracción. Resultados: El grupo de grabado ácido previo por 10 segundos mostró una mayor resistencia a la microtracción con una resistencia mínima de 10,43 MPa y

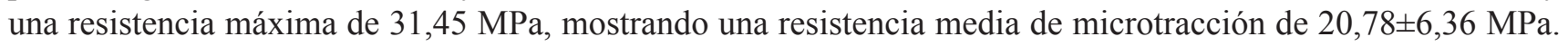
Conclusiones: La resistencia adhesiva a la microtracción en dentina de bovino bajo el modo grabado y enjuague por 10 segundos previo al sistema adhesivo universal Clearfil Universal Bond fue mayor en comparación al grupo tratado solo con el sistema adhesivo universal, además de probar que la exposición por un tiempo reducido de ácido fosfórico en dentina es idóneo para evitar el deterioro de la superficie dentinaria, considerando su morfología.

PALABRAS CLAVE: adhesivo universal, dentina, bovino, microtracción.

Departamento de Clínica Estomatológica, Facultad de Estomatología Roberto Beltrán, Universidad Peruana Cayetano Heredia. Lima, Perú. Facultad de Estomatología Roberto Beltrán, Universidad Peruana Cayetano Heredia. Lima, Perú.

Mg. PhD Profesor Principal

Estudiante de Estomatología 


\section{SUMMARY}

Universal adhesives and their versatility with adhesive techniques generate a controversy between the use and non-use of etching acid before the application of the universal adhesive. Objective: To find the optimal acid etching time prior to the application of Clearfil Universal Bond on bovine tooth dentin, by analyzing the values of resistance to microtraction. Material and methods: Experimental, prospective and comparative study. 6 bovine upper central incisors were used, from which 80 specimens were obtained and divided into 4 groups of 20 specimens each. After complying with the parameters of each group, the specimens were subjected to the microtraction test. Results: The group of acid etching for 10 seconds showed a higher resistance to microtraction with a minimum resistance of $10.43 \mathrm{MPa}$ and a maximum resistance of $31.45 \mathrm{MPa}$, showing an average resistance of microtraction of $20.78 \pm 6.36 \mathrm{MPa}$. Conclusion: The adhesive resistance to microtraction in bovine dentin under the etching and rinsing mode for 10 seconds prior to the Clearfil Universal Bond universal adhesive system was higher compared to the group treated only with the universal adhesive system, in addition to proving that a reduced exposure time of phosphoric acid in dentin is ideal to avoid deterioration of the dentin surface, considering its morphology.

KEY WORDS: universal adhesive, dentin, bovine, microtraction.

\section{INTRODUCCIÓN}

Con la introducción de los sistemas adhesivos universales, lo cuales son populares por la reducción del tiempo clínico y su versatilidad con el modo grabado y enjuague (ER) y el modo de autograbado (SE), se genera controversia sobre la técnica ideal para lograr una mejor adhesión en dentina.

La adhesión lograda en dentina es inestable por su composición orgánica e inorgánica, 70\% hidroxiapatita, $18 \%$ agua y $12 \%$ de colágeno con una alta concentración del tipo I en un $90 \%$, orientado en forma de red $(1,2)$.

La estructura dentinaria es altamente permeable y su exposición prologada al AF puede deteriorar la interfase adhesiva por exposición de las metaloproteinasas que son responsables de la pérdida de colágeno y de la continuidad de la capa híbrida, también por la incompleta penetración de los monómeros de resina en la profundidad de la desmineralización de la dentina, dejando una hibridación incompleta, además de fibrilla colágenas desprotegidas y generar como efecto secundario la hipersensibilidad postoperatoria, por el ensanchamiento de los túbulos dentinarios, llegando cerca de la pulpa $(3,4)$. Erhardt et al., en el 2004 mencionan que el grabado excesivo de la superficie dentinaria podría desnaturalizar la red de colágeno deteriorando la interfase adhesiva y disminuyendo la adhesión (5). En el 2016 Takamizawa et al., determinaron la capacidad de unión a dentina de tres sistemas adhesivos universales con y sin grabado ácido previo, resultando que el grupo tratado con grabado ácido previo al sistema adhesivo universal mostró una resistencia adhesiva menor en comparación al grupo tratado solo con el adhesivo universal (6). Estos estudios establecen que el grabado con AF previo al sistema universal debilita o destruye la interfase adhesiva en dentina, por el riesgo del grabado excesivo $(7,8)$.

El modo de autograbado (SE), simplifica la adhesión a la dentina para evitar el grabado con AF, desmineralizando parcialmente la superficie dentinaria y logrando la unión química mediante la incorporación de monómeros con un nivel de acidez entre leve a moderada, que se comportan como aditivos en la hidroxiapatita restante. Sin embargo, durante la remoción del tejido careado, la superficie dentinaria es manipulada con instrumentos rotatorios o de forma manual quedando como residuo smear layer, que al quedar disperso por toda la superficie dificulta la adhesión, siendo necesaria su remoción bajo el protocolo acondicionamiento convencional, es decir con AF $(9,10)$

Durante la introducción de los sistemas adhesivos universales Ferrari et al., (11) comprobaron su desempeño, señalando que estos sistemas logran la formación de la capa híbrida en menor tiempo, pero delgada y poco consistente. Ogata et al., (12) sostuvieron que elAF cuenta con un $\mathrm{pHde} 0,6$, logrando 
formar una capa híbrida gruesa y resistente, mientras que los sistemas adhesivos autoacondicionantes con la incorporación de los monómeros ácidos, cuentan con $\mathrm{pH}$ básico de 2,0, formando una capa híbrida delgada y menos resistente.

El objetivo del presente trabajo fue evaluar el tiempo óptimo de grabado ácido previo a la aplicación del sistema adhesivo universal Clearfil Universal Bond (CSE/Kuraray América) para lograr una adhesión estable en dentina de dientes de bovino, mediante el análisis de los valores de resistencia a la microtracción.

\section{MATERIAL Y MÉTODOS}

El presente estudio fue de tipo experimental, prospectivo y comparativo.

Se utilizaron 2 maxilas de bovino recién sacrificados para consumo humano, de donde se usaron 6 incisivos centrales superiores de bovino, de los que se obtuvo 80 especímenes separados en 4 grupos de 20 especímenes cada uno de aproximadamente $1 \mathrm{~mm}$ x $1 \mathrm{~mm}$ y $8 \mathrm{~mm}$ de longitud para cada grupo, cortados a nivel de unión cemento adamantino.

Para la selección de las muestras, se usaron los siguientes criterios:

- Criterios de Inclusión: Incisivos de bovino libres de caries y fracturas.

- Criterios de Exclusión: Incisivos de bovino con una corona atrofiada, de menor tamaño, fracturada o con caries.

Comenzamos con la obtención y preparación de muestras y especímenes:

Se obtuvieron 2 maxilas de bovino, de donde se extrajeron cuidadosamente 6 incisivos superiores sanos.

Luego de limpiarlos bien se realizó un corte con discos diamantados a nivel del cuello cervical, separando la corona de la raíz para eliminar el tejido pulpar.

Después se lavó y secó la cámara pulpar y se la rellenó con resina fluida Wave (SDI, Melbourne, Australia).
Posteriormente se desgastó la superficie vestibular con lijas de agua de 220, 400 y 600 exponiendo la superficie dentinaria.

\section{Preparación de la muestra}

En el anexo 1 se muestra el cuadro de operacionalización de variables, en el anexo 2 se muestra la composición química de los materiales probados en este estudio

\section{ler grupo: sin grabado ácido}

Se realizó una profilaxis con el uso de una escobilla profiláctica mezclando piedra pómez más agua por 10 segundos, se lavó con agua de jeringa triple durante 10 segundos, luego se secó con aire de la jeringa triple por 5 segundos, el exceso de agua se absorbió con una torunda de algodón por 2 segundos.

Se dispenso 2 gotas del Clearfil Universal Bond (CSE/Kuraray América) (después del dispensado del adhesivo se volvió a tapar inmediatamente, para que el disolvente no se evapore) y se aplicó dos capas separadas del adhesivo universal, se frotó la preparación con una microbrocha (TPC, tamaño medio, color morado) por 15 segundos, en cada capa. No se fotopolimerizó entre capas, el exceso del disolvente se evaporó secando por completo con aire con una jeringa triple por 10 segundos; antes de polimerizar se verificó que la superficie tenga una aspecto brillante y uniforme. Finalmente, se fotopolimerizó por 10 segundos con una lámpara de luz halógena (Litex 680-Dentamerica-CoronaCalifornia-Usa-A137760), la cual al ser evaluada con un radiómetro tiene una intensidad de luz de 600 $\mathrm{mW} / \mathrm{mm}^{2}$.

\section{2do grupo: con grabado ácido por 10 segundos}

Se realizó una profilaxis con el uso de una escobilla profiláctica mezclando piedra pómez más agua por 10 segundos, se lavó con agua de jeringa triple durante 10 segundos, luego se secó con aire de la jeringa triple por 5 segundos, el exceso de agua se absorbió con una torunda de algodón por 2 segundos.

Se procedió a grabar la superficie de la dentina con ácido fosfórico al 37\% (DENTSPLY) durante unos 10 segundos, se lavó con jeringa triple por 15 segundos para eliminar el ácido y el exceso de humedad fue 
Anexo 1.Cuadro de operacionalización de variables

\begin{tabular}{|c|c|c|c|c|c|c|c|}
\hline & ariable & $\begin{array}{l}\text { Definición } \\
\text { conceptual }\end{array}$ & $\begin{array}{c}\text { Definición } \\
\text { operacional }\end{array}$ & Indicador & $\begin{array}{c}\text { Tipo de } \\
\text { variable }\end{array}$ & $\begin{array}{l}\text { Escala de } \\
\text { medición }\end{array}$ & Valor \\
\hline $\begin{array}{c}\text { Variables } \\
\text { Independiente }\end{array}$ & $\begin{array}{c}\text { Tiempo de } \\
\text { acondicionamiento } \\
\text { ácido }\end{array}$ & $\begin{array}{c}\text { La aplicación } \\
\text { de ácido } \\
\text { fosfórico sobre el } \\
\text { esmalte/dentina } \\
\text { elimina las } \\
\text { irregularidades, } \\
\text { remueve la } \\
\text { contaminación } \\
\text { y permeabiliza } \\
\text { los túbulos } \\
\text { dentinarios, } \\
\text { formando } \\
\text { microporosidades } \\
\text { que vuelven } \\
\text { retentiva a } \\
\text { la superficie } \\
\text { dentaria. }\end{array}$ & $\begin{array}{c}\text { El tiempo de } \\
\text { grabado ácido } \\
\text { determina la } \\
\text { profundidad de } \\
\text { desmineralización } \\
\text { sobre el esmalte/ } \\
\text { dentina. }\end{array}$ & Segundos & Cualitativa & Nominal & $\begin{array}{c}0 \text { segundos } \\
10 \text { segundos } \\
20 \text { segundos } \\
30 \text { segundos }\end{array}$ \\
\hline $\begin{array}{c}\text { Variable } \\
\text { Dependiente }\end{array}$ & $\begin{array}{l}\text { Resistencia a la } \\
\text { microtracción }\end{array}$ & $\begin{array}{l}\text { Es esfuerzo } \\
\text { máximo que } \\
\text { ejerce el } \\
\text { espécimen a la } \\
\text { microtracción, } \\
\text { sin separarse. }\end{array}$ & $\begin{array}{l}\text { Medida de la } \\
\text { resistencia del } \\
\text { espécimen a } \\
\text { la fuerza de } \\
\text { microtracción } \\
\text { ejercida por el } \\
\text { microtensiómetro. }\end{array}$ & $\mathrm{MPa}$ & Cuantitativa & Razón & $0-\infty$ \\
\hline
\end{tabular}

retirado con aire de la misma jeringa triple sin desecar por 5 segundos.

Se dispenso 2 gotas del adhesivo Clearfil Universal Bond(CSE/KurarayAmérica) (después del dispensado del adhesivo se volvió a tapar inmediatamente, para que el disolvente no se evapore) y se aplicó dos capas separadas de adhesivo Clearfil Universal Bond (CSE/ Kuraray América), se frotó la preparación con una microbrocha (TPC, tamaño medio, color morado) por 15 segundos, en cada capa. No se fotopolimerizó entre capas, el exceso del disolvente se evaporó secando por completo con aire con una jeringa triple por 10 segundos; antes de polimerizar se verificó que la superficie tenga una aspecto brillante y uniforme. Finalmente, se fotopolimerizó por 10 segundos con una lámpara de luz halógena (Litex 680-DentamericaCorona-California-Usa-A137760), la cual al ser evaluada con un radiómetro tiene una intensidad de luz de $600 \mathrm{~mW} / \mathrm{mm}^{2}$.

\section{3er grupo: con grabado ácido por 20 segundos}

Se realizó una profilaxis con el uso de una escobilla profiláctica mezclando piedra pómez más agua por 10 segundos, se lavó con agua de jeringa triple durante 10 segundos, luego se secó con aire de la jeringa triple por 5 segundos, el exceso de agua se absorbió con una torunda de algodón por 2 segundos.

Se procedió a grabar la superficie de la dentina con ácido fosfórico al 37\% (DENTSPLY) durante unos 20 segundos, se lavó con jeringa triple por 15 segundos para eliminar el ácido y el exceso de humedad fue retirado con aire de la misma jeringa triple sin desecar por 5 segundos.

Se dispenso 2 gotas del adhesivo Clearfil Universal Bond (CSE/Kuraray América) (después del dispensado del adhesivo se volvió a tapar inmediatamente, para que el disolvente no se evapore) 
Anexo 2. Cuadro de composición química de los materiales probados en este estudio

\begin{tabular}{|c|c|c|c|}
\hline Material & Composición & pH & Instrucciones del fabricante \\
\hline $\begin{array}{l}\text { Acondicionador dental } \\
\text { en gel (DENTSPLY) } \\
\text { LOTE: } 107220 \mathrm{H}\end{array}$ & $\begin{array}{l}37 \% \text { de ácido fosfórico, sílice } \\
\text { coloidal, agua y colorante }\end{array}$ & 0.6 & 1. Aplicar y dejar durante 30 segundos. \\
\hline $\begin{array}{l}\text { Clearfil Universal Bond } \\
\text { LOTE: 4Q0014 }\end{array}$ & $\begin{array}{l}\text { 10-MDP, Bis-GMA, HEMA, } \\
\text { hidrofílico monómero de } \\
\text { amida, sílice coloidal, etanol,dl- } \\
\text { canforquinona, aceleradores, agua } \\
\text { y fluoruro de sodio. }\end{array}$ & 2.3 & $\begin{array}{l}\text { 1. Aplicar BOND (fricción de } 10 \mathrm{seg} \text { ) } \\
\text { 2. Secar con flujo de aire a presión suave por } 5 \mathrm{seg} \\
\text { 3. Fotopolimerizar durante } 10 \mathrm{seg}\end{array}$ \\
\hline
\end{tabular}

Anexo 3. Ficha de recolección de datos para los grupos control

\begin{tabular}{|c|c|c|c|c|c|c|}
\hline \multicolumn{7}{|c|}{ CONTROL } \\
\hline $\begin{array}{l}\text { Sistema } \\
\text { Adhesivo } \\
\text { Universal }\end{array}$ & Especímenes & $\begin{array}{l}\text { Área } \\
\text { Ancho }\end{array}$ & Espesor & Total & $\begin{array}{l}\text { Fuerza de } \\
\text { microtracción en } N\end{array}$ & $\begin{array}{l}\text { Fuerza de resistencia a la } \\
\text { microtracción en MPa }\end{array}$ \\
\hline \multirow{20}{*}{ 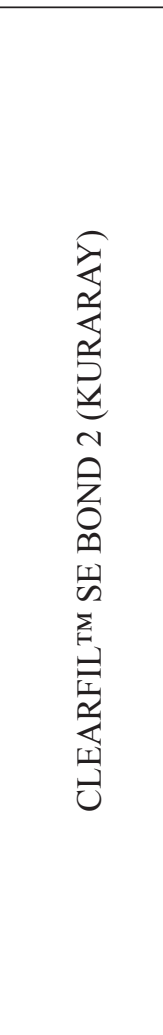 } & 1 & & & & & \\
\hline & 2 & & & & & \\
\hline & 3 & & & & & \\
\hline & 4 & & & & & \\
\hline & 5 & & & & & \\
\hline & 6 & & & & & \\
\hline & 7 & & & & & \\
\hline & 8 & & & & & \\
\hline & 9 & & & & & \\
\hline & 10 & & & & & \\
\hline & 11 & & & & & \\
\hline & 12 & & & & & \\
\hline & 13 & & & & & \\
\hline & 14 & & & & & \\
\hline & 15 & & & & & \\
\hline & 16 & & & & & \\
\hline & 17 & & & & & \\
\hline & 18 & & & & & \\
\hline & 19 & & & & & \\
\hline & 20 & & & & & \\
\hline
\end{tabular}

y se aplicó dos capas separadas de adhesivo universal, se frotó la preparación con una microbrocha (TPC, tamaño medio, color morado) por 15 segundos, en cada capa. No se fotopolimerizó entre capas, el exceso del disolvente se evaporó secando por completo con aire con una jeringa triple por 10 segundos; antes de polimerizar se verificó que la superficie tenga una aspecto brillante y uniforme. Finalmente, se fotopolimerizó por 10 segundos con una lámpara de luz halógena (Litex 680-Dentamerica-Corona-
California-Usa-A137760), la cual al ser evaluada con un radiómetro tiene una intensidad de luz de 600 $\mathrm{mW} / \mathrm{mm}^{2}$.

\section{4to grupo: con grabado ácido por 30 segundos}

Se realizó una profilaxis con el uso de una escobilla profiláctica mezclando piedra pómez más agua por 10 segundos, se lavó con agua de jeringa triple durante 10 segundos, luego se secó con aire de la jeringa triple 
Anexo 4. Fichas de recolección de datos para los grupos con 10 segundos de acondicionamiento previo.

\begin{tabular}{|c|c|c|c|c|c|c|}
\hline \multicolumn{7}{|c|}{10 SEGUNDOS DE ACONDICIONADO } \\
\hline $\begin{array}{l}\text { Sistema } \\
\text { Adhesivo } \\
\text { Universal }\end{array}$ & Especímenes & $\begin{array}{l}\text { Área } \\
\text { Ancho }\end{array}$ & Espesor & Total & $\begin{array}{l}\text { Fuerza de } \\
\text { microtracción en } \mathrm{N}\end{array}$ & $\begin{array}{l}\text { Fuerza de resistencia a la } \\
\text { microtracción en MPa }\end{array}$ \\
\hline 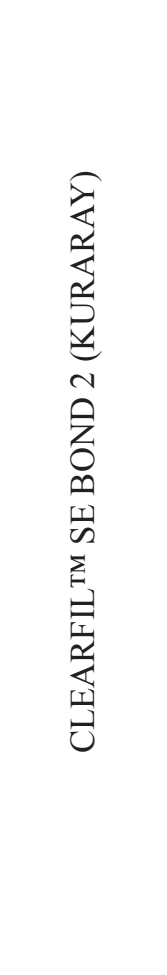 & $\begin{array}{l}2 \\
3 \\
4 \\
5 \\
6 \\
7 \\
8 \\
9 \\
10 \\
11 \\
12 \\
13 \\
14 \\
15 \\
16 \\
17 \\
18 \\
19 \\
20\end{array}$ & & & & & \\
\hline
\end{tabular}

por 5 segundos, el exceso de agua se absorbió con una torunda de algodón por 2 segundos.

Se procedió a grabar la superficie de la dentina con ácido fosfórico al 37\% (DENTSPLY) durante unos 30 segundos, se lavó con jeringa triple por 15 segundos para eliminar el ácido y el exceso de humedad fue retirado con aire de la misma jeringa triple sin desecar por 5 segundos.

Se dispenso 2 gotas del adhesivo Clearfil Universal Bond (CSE/Kuraray América) (después del dispensado del adhesivo se volvió a tapar inmediatamente, para que el disolvente no se evapore) y se aplicó dos capas separadas de adhesivo universal, se frotó la preparación con una microbrocha (TPC, tamaño medio, color morado) por 15 segundos, en cada capa. No se fotopolimerizó entre capas, el exceso del disolvente se evaporó secando por completo con aire con una jeringa triple por 10 segundos; antes de polimerizar se verificó que la superficie tenga una aspecto brillante y uniforme. Finalmente, se fotopolimerizó por 10 segundos con una lámpara de luz halógena (Litex 680-Dentamerica-CoronaCalifornia-Usa-A137760), la cual al ser evaluada con un radiómetro tiene una intensidad de luz de 600 $\mathrm{mW} / \mathrm{mm}^{2}$.

Para terminar, en todos los grupos con ayuda de anillos de $8 \mathrm{~mm}$ de diámetro y un grosor de $4 \mathrm{~mm}$, se aplicó la resina de nanorelleno Filtek ${ }^{\mathrm{TM}}$ Z250 (3M ESPE, MN, USA) con la técnica incremental y fotopolimerizada con la luz halógena (Litex 680-Dentamerica-Corona-California-Usa-A137760) Luego fueron almacenados en agua destilada por 24horas (figura 1).

\section{Cortes para la evaluación}

Fue necesario el uso de cera vidriosa para fijar la muestra a una platina de acrílico, esto se realizó derritiendo un poco del material con ayuda de un mechero, luego fue colocado sobre la platina y sobre 
Anexo 5. Fichas de recolección de datos para los grupos con 20 segundos de acondicionamiento previo.

\begin{tabular}{|c|c|c|c|c|c|c|}
\hline \multicolumn{7}{|c|}{20 SEGUNDOS DE ACONDICIONADO } \\
\hline $\begin{array}{l}\text { Sistema } \\
\text { Adhesivo } \\
\text { Universal }\end{array}$ & Especímenes & $\begin{array}{l}\text { Área } \\
\text { Ancho }\end{array}$ & Espesor & Total & $\begin{array}{l}\text { Fuerza de } \\
\text { microtracción en } N\end{array}$ & $\begin{array}{l}\text { Fuerza de resistencia a la } \\
\text { microtracción en MPa }\end{array}$ \\
\hline \multirow{20}{*}{ 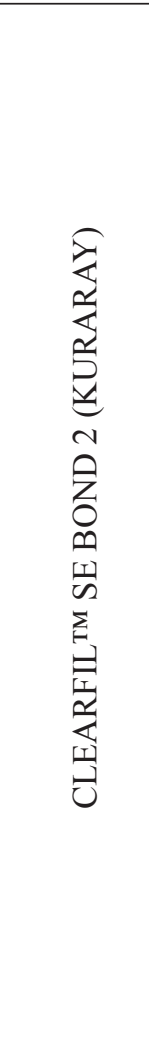 } & 1 & & & & & \\
\hline & 2 & & & & & \\
\hline & 3 & & & & & \\
\hline & 4 & & & & & \\
\hline & 5 & & & & & \\
\hline & 6 & & & & & \\
\hline & 7 & & & & & \\
\hline & 8 & & & & & \\
\hline & 9 & & & & & \\
\hline & 10 & & & & & \\
\hline & 11 & & & & & \\
\hline & 12 & & & & & \\
\hline & 13 & & & & & \\
\hline & 14 & & & & & \\
\hline & 15 & & & & & \\
\hline & 16 & & & & & \\
\hline & 17 & & & & & \\
\hline & 18 & & & & & \\
\hline & 19 & & & & & \\
\hline & 20 & & & & & \\
\hline
\end{tabular}

ella la muestra a fijar, luego pasamos a realizar los cortes seriados de forma horizontal y vertical por medio de una máquina de corte (figura 2), obteniendo especímenes de aproximadamente $1 \times 1 \mathrm{~mm}$ y $8 \mathrm{~mm}$ de longitud, 20 por grupo.

\section{Prueba de microtracción}

Fue realizada en el grupo control y en los grupos en los que se anticipará el acondicionado con ácido fosfórico al $37 \%$ por un promedio de 10,20 y 30 segundos respectivamente (Anexo 3, anexo 4, anexo 5 y anexo 6). La velocidad a la cual será medida la resistencia a la microtracción será de $0,5 \mathrm{~mm} / \mathrm{min}$ con la máquina de microtensión (Bisco, Schaumburg, IL) (figura 3).

Este estudio fue ejecutado luego de recibir una aprobación de la Unidad Integrada de Gestión de Investigación, Ciencia y Tecnología de las Facultades de Medicina, de Estomatología y de Enfermería, con el código de SIDISI N 101700.

Debido a que la naturaleza del trabajo de investigación es experimental y para su realización se utilizarán dientes de bovino, pasó por el Comité Institucional de Ética para Animales (CIEA-UPCH) de la Universidad Peruana Cayetano Heredia, el cual otorgó la exoneración para su ejecución.

Los datos fueron procesados en el programa estadístico Stata/SE15.0.

Los valores de resistencia a la tracción expresados en Newton, fueron convertidos a Megapascales y registrados en una ficha de recolección de datos por grupo.

De ellos se obtuvieron promedios, mediana, y desviación estándar, para luego someter a cada grupo a la prueba de Shapiro Wilk, para determinar su distribución. 
Anexo 6. Fichas de recolección de datos para los grupos con 30 segundos de acondicionamiento previo.

\begin{tabular}{|c|c|c|c|c|c|c|}
\hline \multicolumn{7}{|c|}{30 SEGUNDOS DE ACONDICIONADO } \\
\hline $\begin{array}{l}\text { Sistema } \\
\text { Adhesivo } \\
\text { Universal }\end{array}$ & Especímenes & $\begin{array}{l}\text { Área } \\
\text { Ancho }\end{array}$ & Espesor & Total & $\begin{array}{l}\text { Fuerza de } \\
\text { microtracción en } \mathbf{N}\end{array}$ & $\begin{array}{l}\text { Fuerza de resistencia a la } \\
\text { microtracción en MPa }\end{array}$ \\
\hline \multirow{20}{*}{ 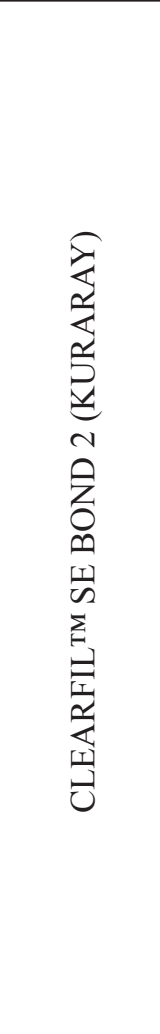 } & 1 & & & & & \\
\hline & 2 & & & & & \\
\hline & 3 & & & & & \\
\hline & 4 & & & & & \\
\hline & 5 & & & & & \\
\hline & 6 & & & & & \\
\hline & 7 & & & & & \\
\hline & 8 & & & & & \\
\hline & 9 & & & & & \\
\hline & 10 & & & & & \\
\hline & 11 & & & & & \\
\hline & 12 & & & & & \\
\hline & 13 & & & & & \\
\hline & 14 & & & & & \\
\hline & 15 & & & & & \\
\hline & 16 & & & & & \\
\hline & 17 & & & & & \\
\hline & 18 & & & & & \\
\hline & 19 & & & & & \\
\hline & 20 & & & & & \\
\hline
\end{tabular}

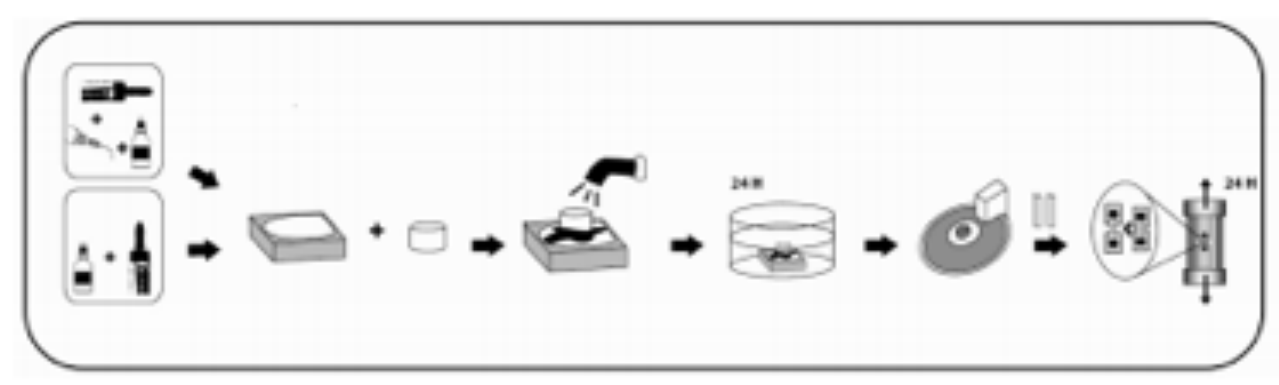

Figura 1. Procedimiento experimental.

Los valores de resistencia a la microtracción de los diferentes tiempos de acondicionamiento previo fueron evaluados bajo la prueba de ANOVA. También se los sometió a la prueba de Post hoc (Scheffe) para una evaluación más específica.

\section{RESULTADOS}

Se realizó un estudio es de tipo experimental, prospectivo y comparativo in vitro con el propósito de encontrar el tiempo óptimo de grabado ácido previo a la aplicación del adhesivo Clearfil Universal Bond sobre la dentina de bovino, mediante el análisis de los valores de resistencia a la microtracción.

Para la evaluación se formaron 4 grupos, uno control el cual se trabajó solo con el adhesivo Clearfil Universal Bond y los otros grupos bajo el modo ER por el tiempo de 10, 20 y 30 segundos previo al sistema adhesivo universal con variaciones de tiempo, encontrándose los siguientes resultados utilizando las abreviaturas y símbolos definidos en el anexo 7 : 


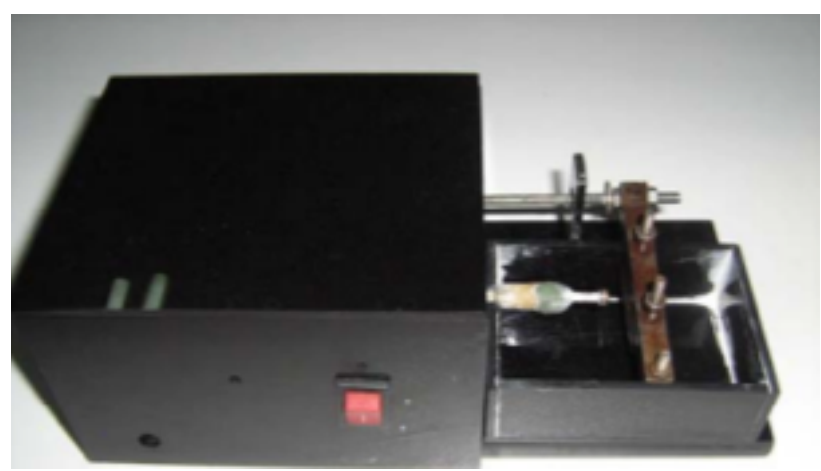

Figura 2. Máquina de corte.

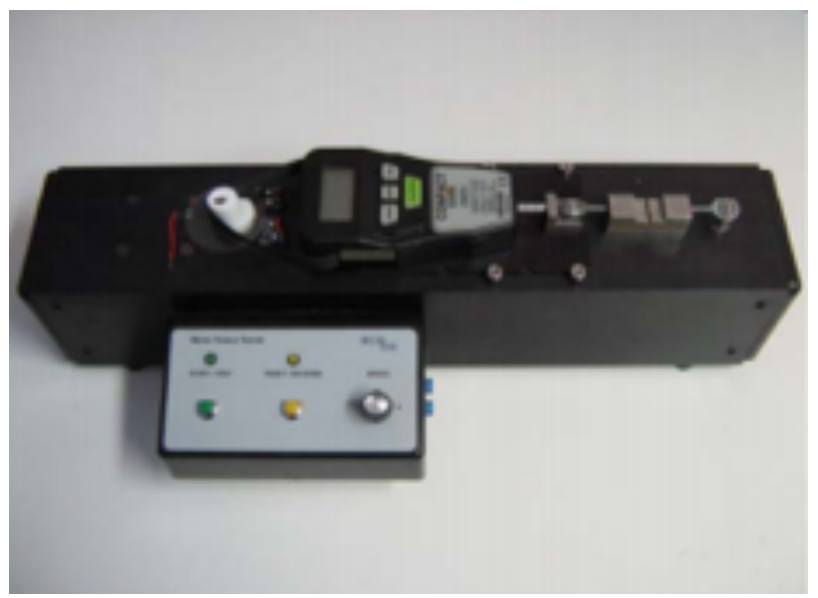

Figura 3. Microtensiómetro Microtensile Tester (BISCO, EE. UU)

Anexo 7. Lista de abreviaturas y símbolos

\begin{tabular}{|c|c|c|}
\hline$\mu \mathrm{TBS}$ & $:$ & Microtensión \\
\hline 10-MDP & : & 10-metacriloiloxidocil dihidrofosfato. \\
\hline ER & : & Modo grabado y enjuague (Etch \& Rinse) \\
\hline SE & : & Modo autograbado (Self-etch) \\
\hline $\mathbf{A F}$ & : & Ácido fosfórico. \\
\hline HEMA & : & Metacrilato de 2-hidroxietilo. \\
\hline TAGS & : & Monómero de resina. \\
\hline S. E. M. & : & Microscopia electrónica de barrido. \\
\hline$\mu \mathrm{m}$ & : & Micrómetros. \\
\hline MPa & : & Megapascales. \\
\hline $\mathbf{N}$ & : & Newton. \\
\hline
\end{tabular}

La media de la resistencia a la microtracción ( $\mu$ TBS) del grupo sin tratamiento acido fue de $14,68 \pm 4,8 \mathrm{MPa}$. Mientras que para los grupos bajo el modo ER por el tiempo de 10, 20 y 30 segundos, se encontraron valores de $20,78 \pm 6,36 \mathrm{MPa}$, $19,02 \pm 4,46 \mathrm{MPa}$ y $15,91 \pm 3,63 \mathrm{MPa}$ respectivamente.
Los cuatro grupos fueron sometidos a la prueba estadística de Shapiro Wilk para determinar su distribución, resultando una distribución normal $(\mathrm{p}>0,05)$. Seguida de la prueba ANOVA, donde se observa que existe una diferencia estadísticamente significativa, en al menos un par de grupos (valor $\mathrm{p}=0,0006)$ (tabla 1$)$.

Para obtener un resultado más específico sobre la diferencia entre los grupos, se realiza una comparación múltiple de valores, mediante la prueba estadística de Post hoc (scheffe), encontrando diferencias significativas entre el grupo bajo el modo ER por 10 segundos y el grupo control (valor $\mathrm{p}=0,003$ ) y el grupo bajo el modo ER por el tiempo de 30 segundos (valor $\mathrm{p}=0,026) \mathrm{p}>0,05$ (tabla 2).

\section{DISCUSIÓN}

En este estudio se utilizaron dientes de bovino como sustituto de dentina humana, su fácil obtención y su poca o ninguna diferencia tanto a nivel macro como microscópico con respecto a los dientes humanos, los convierten en un excelente sustrato para realizar estudios. $(11,16)$. La prueba de microtracción es usada para evaluar fallas locales, exclusivas de la interfase adhesiva, ya sean adhesivas o cohesivas, lo que permite un análisis real de la resistencia de unión entre el material y la estructura dentaria (17).

Según los resultados del estudio encontramos diferencias significativas para el grupo bajo el modo ER por 30 segundos frente al grupo bajo el modo ER por 10 segundos, en donde muestra una resistencia de unión significativamente más baja a la prueba de $\mu$ TBS $(p=0,026)$. Se puede decir entonces que el grupo bajo el modo ER por el tiempo de 30 segundos creó una interfaz de enlace inferior entre el adhesivo universal y la dentina. Ahora como se aprecia para los grupos bajo el modo ER en los tiempos de 10 y 20 segundos no hubo diferencia estadística, sin embargo, si comparamos las fuerzas de unión logradas en ambos tiempos, vemos que la diferencia es de $1,76 \mathrm{MPa}$, lo que resulta en una diferencia casi significativa en la comparación bajo la prueba Scheffe para el grupo control y el de grabado por 20 segundos (valor $\mathrm{p}=0,06)$.

La principal preocupación que existe sobre el uso de $\mathrm{AF}$ en dentina es que puede disolver la capa frotis 
Tabla 1. Comparación de la resistencia a la microtracción entre el grupo control y los grupos bajo el modo ER por los tiempos de 10, 20 y 30 segundos.

\begin{tabular}{lcccccc}
\hline \multicolumn{2}{l}{ Tiempo acondicionado } & Mínimo & Máximo & Promedio & D.E. & Normalidad \\
\hline Grupo Control (A) & $7,49^{*}$ & $22,87^{*}$ & $14,68^{*}$ & 4,88 & 0,13 \\
10 segundos & (B) & $10,43^{*}$ & $31,45^{*}$ & $20,78^{*}$ & 6,36 & 0,82 \\
20 segundos & (C) & $10,08^{*}$ & $28,20^{*}$ & $19,02^{*}$ & 4,46 & 0,99 \\
30 segundos & (D) & $9,32 *$ & $21,59^{*}$ & $15,91^{*}$ & 3,63 & 0,20 \\
Prueba ANOVA un factor & & & & & $\mathrm{p}=0,0006$ \\
\hline
\end{tabular}

Diferencias significativas encontradas entre los grupos (A-B y B-D) $p<0,05$. Prueba de comparaciones múltiples Scheffe

${ }^{*} \mathrm{MPa}$

Tabla 2. Prueba Scheffe para la comparación de la resistencia a la microtracción para el grupo control y los grupos bajo el modo ER por los tiempos de 10, 20 y 30 segundos.

\begin{tabular}{cccc}
\hline Col Mean & Sin grabado & 10seg de grabado & 20seg de grabado \\
\hline 10seg gr & $6,10^{*}$ & & \\
& 0,003 & & \\
20seg gr & $4,34^{*}$ & $1,76^{*}$ & \\
& 0,06 & 0,735 & $3,10^{*}$ \\
30seg gr & $1,24^{*}$ & $4,86^{*}$ & 0,274 \\
\hline
\end{tabular}

* Mpa

que cubre la dentina en su totalidad y desmineralizar completamente la hidroxiapatita, exponiendo a las mallas de colágeno a la degradación hidrolítica, obstaculizando la formación de la capa híbrida (10, 20). Sato et al., probaron el efecto de otros ácidos sobre la superficie dentinaria, todos con un nivel de $\mathrm{pH}$ similar al AF y bajo el tiempo de tratamiento de 15 segundos (21). A la evaluación con SEM de los diferentes tratamientos observó que la superficie dentinaria tratada con AF mostraba la red de colágeno expuesta, mientras que los demás tratamientos mostraron una cantidad mínima de colágeno y túbulos dentinarios parcialmente desmineralizados. Frente a la prueba $\mu$ TBS se muestra que la resistencia de unión lograda por la superficie tratada con AF mostró una menor resistencia frente a los demás tratamientos, sin embargo, ninguno mostró una diferencia significativa frente al grupo control. La concentración usada de AF en el estudio fue el $40 \%$ con un $\mathrm{pH} 0,6$ por un tiempo de 15 segundos, frente a los resultados presentados, se concluye que el tiempo de exposición del AF a dentina es determinante, pero también lo es su concentración. Según los resultados presentados que el uso del AF al 37\% por el tiempo de 10 segundos no afecta negativamente la fuerza de unión de la dentina, al contrario, la refuerza.

Siguiendo con los resultados encontrados tenemos que el grupo bajo el modo ER por el tiempo de 10 segundos previo al uso del adhesivo universal aumenta significativamente la fuerza de resistencia a la $\mu$ TBS frente al grupo control (valor $\mathrm{p}=0,003$ ).

El adhesivouniversalClearfil Universal Bondutilizado en este estudio contiene dos componentes principales que logran la unión a dentina mediante enlaces químicos, estos son 10-MDP y HEMA. 10-MDP es un monómero de fosfato que se une iónicamente con los cristales de hidroxiapatita ubicados alrededor del colágeno, formando nanocapas sobre las que hay una deposición continua de estos monómeros, procedimiento conocido como "nanolayering". Esta unión química es estable en agua, lo que también nos da la idea de una unión estable a largo plazo. Según las pruebas $\mu \mathrm{TBS}$ realizadas los adhesivos universales que contienen este componente mostraron valores más altos en la fuerza de unión (22). 
En cuanto al monómero hidrofílico HEMA, mejora la fuerza de unión en dentina porque pueden penetrar profundamente la dentina desmineralizada, polimerizar y desarrollar una red de polímeros estable que produzca fuerte retención micromecánica. Kuno et al., probaron esa afirmación sometiendo a diferentes adhesivos universales a la prueba $\mu \mathrm{TBS}$, determinando que la mayor resistencia de unión fue para los adhesivos que dentro de su composición presentaban al monómero HEMA, bajo un tiempo de aplicación extendido (23).

Saito et al., evaluaron la fuerza de unión lograda para los modos ER y SE en adhesivos universales y sometiendo las muestras a la al análisis con SEM (24). El autor encontró una clara diferencia sobre los diferentes modos de grabado, para el modo SE encontró una desmineralización pobre y parte de la capa frotis disuelta, en cuanto al modo ER la capa frotis estaba disuelta y se observaron lo túbulos dentinarios abiertos. Al observar la interfase adhesiva encontró que en el modo SE los tags de resina infiltrados eran escasos, aquí el grupo con el tiempo de aplicación prolongado mostró tags de resina ligeramente más largos, sin embargo, para el modo ER se observó que los tags de resina presentaban una longitud mayor en los túbulos dentinarios, igual que en el otro modo de aplicación los que tuvieron un tiempo más prologado mostraron una mayor longitud. Llegando a la conclusión que es necesaria una interacción física como también química para lograr una fuerza de adhesión exitosa y que el mejor modo de lograrlo es someter a la superficie dentinaria al tratamiento con el modo ER y SE denominado por la literatura como "multimodo" (25).

Algunos estudios plantean que el nivel de acidez del adhesivo universal tiene efectos potenciales en las propiedades mecánicas a largo plazo y estabilidad de la interfaz adhesiva (26).

Como ejemplo tenemos a Cardoso et al., quienes compararon la fuerza de adhesión en dentina en los modos SE y ER para 5 tipos de adhesivo universal, bajo un régimen de envejecimiento de la interfase adhesiva de 24hr y de 6 meses (27); encontaron que el adhesivo universal que presentó mayor estabilidad a las 24 horas y a los 6 meses fue Ybond Universal con un $\mathrm{pH}$ de 1.4 el bajo el modo ER y el que en promedio tuvo menor fuerza de unión fue G-Bond con un $\mathrm{pH}$ de 2.55 y Tetric-N-Bond Universal con un $\mathrm{pH}$ de 2.95 sin embargo, los resultados no fueron significativos. Indicando que el rendimiento de unión de los adhesivos universales a la dentina es dependiente del material y de su composición. Ahmed et al., midieron la fuerza de unión $\mu$ TBS del adhesivo universal Clearfil Universal Bond Quick bajo los modos SE y ER en comparación con otros, bajo un régimen de envejecimiento de la interfase adhesiva de 1 semana y de 6 meses (24). Encontraron que el adhesivo universal Clearfil Universal Bond Quick con un $\mathrm{pH}$ de 2,3, presentó valores altos de resistencia de unión a la $\mu$ TBS a la evaluación de una semana, bajo el modo ER y por un tiempo prolongado de aplicación del adhesivo a dentina de 20 segundos, sin embargo, a la evaluación de 6 meses disminuyó. De igual forma los resultados no fueron significativos. E1 adhesivo universal Clearfil Universal Bond utilizado nos ofrece un $\mathrm{pH}$ de 2,3, los estudios presentados indican que en nivel de acidez y composición del adhesivo universal es determinante para lograr una interfase estable a largo plazo, variables que se deben considerarse para futuros estudios.

Hasta aquí nos queda claro que el modo idóneo para lograr una adhesión exitosa a largo plazo en dentina es el "multimodo" que complementa la interacción química con la física.

Según los resultados existe una diferencia casi significativa en la comparación con la prueba Scheffe para el grupo control y el de grabado por 20 segundos (valor $\mathrm{p}=0,06$ ), resultante de la pequeña diferencia entre $\operatorname{los} 10$ y 20 segundos de grabado previo al sistema adhesivo universal, de 1,76MPa.

Debemos recordar que el tiempo es definitivo para el tratamiento de la superficie dentinaria, ya que determinará la degradación de la interfase adhesiva, la cual puede ser hidrolítica o proteolítica, además durante la interacción química, la introducción de los monómeros hidrofílicos ocasiona un aumento en la sorción de agua, lo que con el tiempo produce también una degradación hidrolítica de la interfase adhesiva $(3,4,5,9)$.

Si bien es cierto, los estudios experimentales nos facilitan la evaluación de las propiedades mecánicas de los biomateriales y nos permiten aportar técnicas que mejoran su función, sin embargo, existen 
muchos factores presentes en el ambiente oral que afectan la fuerza de unión de los adhesivos a la estructura del diente, como por ejemplo tenemos la carga masticatoria, alteraciones de $\mathrm{pH}$ y cambios térmicos, diversas condiciones orales que dificultan la predicción sobre el correcto funcionamiento de los adhesivos en el entorno clínico (28). Frente a estas limitaciones se recomiendan ensayos clínicos controlados para emitir mejores resultados.

De la presente investigación se puede concluir que la resistencia adhesiva a la microtracción en dentina de bovino bajo el modo grabado y enjuague por 10 segundos previo al sistema adhesivo universal Clearfil Universal Bond fue mayor en comparación al grupo tratado solo con el sistema adhesivo universal. En cuanto al tiempo de exposición de la dentina al ácido fosfórico, se concluye que un tiempo reducido es el idóneo para evitar el deterioro de la superficie dentinaria, considerando su morfología.

Conflicto de intereses: Los autores no tienen conflictos de interés con este informe.

Aprobación de ética: Este estudio fue ejecutado luego de recibir una aprobación de la Unidad Integrada de Gestión de Investigación, Ciencia y Tecnología de las Facultades de Medicina, de Estomatología y de Enfermería, con el código de SIDISI N 101700.

Debido a que la naturaleza del trabajo de investigación es experimental y para su realización se utilizarán dientes de bovino, pasó por el Comité Institucional de Ética para Animales (CIEA-UPCH) de la Universidad Peruana Cayetano Heredia, el cual otorgó la exoneración para su ejecución.

\section{Financiamiento: Ninguno}

Contribuciones de los autores: Todos los autores contribuyeron en este estudio.

\section{Correspondencia:}

Allison Ruth Bernal Ponce

Jr. Miguel Ortiz 517 - Chalet \#28

Lima, Los Olivos, Perú.

Correo electrónico: allison.bernal.p@upch.pe

\section{REFERENCIAS BIBLIOGRÁFICAS}

1. Masarwa N, Mohamed A, Abou-Rabii I, Abu Zaghlan R, Steier L. Longevity of Self-etch Dentin Bonding Adhesives Compared to Etch-and-rinse Dentin Bonding Adhesives: A Systematic Review. J Evid Based Dent Pract. 2016;16(2):96-106.

2. Fusayama T, Nakamura M, Kurosaki N, Iwaku M. Nonpressure adhesion of adhesive restorative resin. Journal of Dental Research. 1979; 58(4): 1364.

3. Miyazaki M, Tsubota K, Takamizawa T, Kurokawa H, Rikuta A, Ando S. Factors affecting the in vitro performance of dentin-bonding systems. Jpn Dent Sci Rev. 2012; 48: 53-60.

4. Perdigão J, Sezinando A, Monteiro P. Effect of substrate age and adhesive composition on dentin bonding. Oper Dent. 2013; 38 (3): 267-274.

5. Erhardt MCG, Cavalcante LMA, Pimenta LAF. Influence of phosphoric acid pretreatment on selfetching bond strengths. Journal of esthetic and restorative dentistry. $2004 ; 16(1): 33-40$.

6. Takamizawa T, Barkmeier WW, Tsujimoto A, et al. Influence of different etching modes on bond strength and fatigue strength to dentin using universal adhesive systems. Dent Mater. 2016;32(2):e9-21.

7. Mena-Serrano A, Kose C, De Paula EA, et al. A new universal simplified adhesive: 6-month clinical evaluation. J Esthet Restor Dent. 2013; 25(1):55-69.

8. Saker S, Özcan M, Al-Zordk W. The impact of etching time and material on bond strength of self-adhesive resin cement to eroded enamel. Dent Mater J. 2019; 38(6):921-927.

9. Van Landuyt KL, Snauwaert J, De Munck J, et al. Systematic review of the chemical composition of contemporary dental adhesives. Biomaterials . 2007; 28(26):3757-3785.

10. Spencer P, Ye Q, Park J, et al. Adhesive/Dentin Interface: The Weak Link in the composite restoration. Ann Biomed Eng. 2010; 38: 1989- 2003.

11. Ahmed MH, Yoshihara K, Mercelis B, Van Landuyt K, Peumans M, Van Meerbeek B. Quick bonding using a universal adhesive. Clin Oral Investig. 2020; 24(8): 2837-2851.

12. Rüttermann S, Braun A, Janda R. Shear bond strength and fracture analysis of human vs. bovine teeth. PLoS One. 2013; 8(3):e59181.

13. Nakamichi I, Iwaku M, Fusayama T. Bovine teeth as possible substitutes in the adhesion test. Journal of Dental Research. 1983; 62(10): 1076-81.

14. Schilke R, Lisson J, Bauss O, Geurtsen W. Comparison of the number and diameter of dentinal tubules in human and bovine dentine by scanning electron microscopic investigation. Archives Of Oral Biology. 2000; 45(5): 355-61. 
15. Hanabusa M, Mine A, Kuboki T, et al. Bonding effectiveness of a new 'multi-mode' adhesive to enamel and dentine. J Dent. 2012; 40:475-484.

16. Reis A, Giannini M, Kavaguchi A, Soares C, Line S. Comparison of microtensile bond strength to enamel and dentin of human, bovine, and porcine teeth. The Journal Of Adhesive Dentistry. 2004; vol. 6(2): 117-21.

17. Pimentel F, Perlatti P, Suga R, Marins de Carvalho R. Testes mecânicos para a avaliação Laboratorial da união resina/dentina. Rev Fac Odontol Bauru. 2002; 10 (3):118-27.

18. Ferrari M, Davidson C. In vivo resin-dentin interdiffusion and tag formation with lateral branches of two adhesive systems. The Journal Of Prosthetic Dentistry. 1996; 76(3): 250-53.

19. Ogata M, Okuda M, Nakajima M, Pereira P, Sano H, Tagami J. Influence of the direction of tubules on bond strength to dentin. Operative Dentistry. 2001; 26(1): 27-35.

20. Leite MLAES, Costa CAS, Duarte RM, Andrade AKM, Soares DG. Bond strength and cytotoxicity of a universal adhesive according to the hybridization strategies to dentin. Braz Dent J. 2018;29(1):68-75.

21. Sato T, Takagaki T, Baba Y, et al. Effects of different tooth conditioners on the bonding of universal selfetching adhesive to dentin. J Adhes Dent. 2019; 21(1):77-85.

22. Nagarkar S, Theis-Mahon N, Perdigão J. Universal dental adhesives: Current status, laboratory testing, and clinical performance. J Biomed Mater Res B Appl Biomater. 2019; 107(6):2121-2131.
23. Kuno Y, Hosaka K, Nakajima M, et al. Incorporation of a hydrophilic amide monomer into a one-step selfetch adhesive to increase dentin bond strength: Effect of application time. Dent Mater J. 2019;38(6):892-899.

24. Saito T, Takamizawa T, Ishii R, et al. Influence of application time on dentin bond performance in different etching modes of universal adhesives. Oper Dent. 2020;45(2):183-195.

25. Van Meerbeek B, De Munck J, Yoshida Y, et al. Buonocore memorial lecture. Adhesion to enamel and dentin: current status and future challenges. Oper Dent. 2003;28:215-235.

26. Rosa WL, Piva E, Silva AF. Bond strength of universal adhesives: a systematic review and meta-analysis. J Dent. 2015; 43, 765-776.

27. Cardoso GC, Nakanishi L, Isolan CP, Jardim PDS, Moraes RR. Bond stability of universal adhesives applied to dentin using etch-and-rinse or self-etch strategies. Braz Dent J. 2019 ;30(5):467-475.

28. Braga RR, Meira JB, Boaro LC, Xavier TA. Adhesion to tooth structure: a critical review of macro test methods. Dent Mater. 2010; 26(2):e38-49.

Recibido : 14-02-2021

Aceptado : 12-06-2021 